\title{
A Study to Assess the Risk Factors of Thyroid Functions among Bangladeshi Women during 1st Trimester of Pregnancy
}

\section{Anika Bushra ( $\square$ anikabushra8899@gmail.com )}

Department of Biochemistry and Microbiology, North South University, Dhaka, Bangladesh https://orcid.org/0000-0001-5603-075X

\section{Khandaker Sabit Bin Razzak}

Department of Public Health, American International University-Bangladesh, Dhaka, Bangladesh. https://orcid.org/0000-0001-9610-8945

\section{Mohammad Nabil Hossain}

College of Biological and Pharmaceutical Sciences, China Three Gorges University, Hubei, China https://orcid.org/0000-0001-6647-5701

\section{A.S.M. Sarwar}

Topbright https://orcid.org/0000-0002-3315-6496

\section{Research Article}

Keywords: Thyroid functions, Overt Hypothyroidism, Sub-clinical hypothyroidism

Posted Date: August 10th, 2020

DOI: https://doi.org/10.21203/rs.3.rs-49307/v1

License: (9) This work is licensed under a Creative Commons Attribution 4.0 International License. Read Full License 


\section{Abstract}

Background: In recent years many researches has centered on the influence of thyroid disease on pregnancy and the consequence of offspring.

Aim: The present study was conducted to evaluate the relationship of multiple risk factors and thyroid functions among Bangladeshi young women during 1 st half of the pregnancy.

Material and methods:

This cross-sectional study was done in in the Department of Obstetrics \& Gynecology of Dhaka Medical College Hospital, Dhaka on 200 pregnant women in their 1st half of pregnancy attending antenatal clinic, DMCH from December 2018 to December 2019. Where inclusion criteria were healthy singleton pregnant women up to 20 weeks of gestation.

Results:

According to this study, $10.5 \%$ subclinical hypothyroidism group and $36.8 \%$ from overt hypothyroidism patients had family history of thyroid dysfunction. $6.5 \%$ had stage- 1 hypertension and $11 \%$ patients had stage-2 hypertension. Also, abnormal blood pressure level is highly correlated with thyroid dysfunction status. The rate of iron disfunction strongly correlated with thyroid dysfunction. $6 \%$ patients had mid-level of iron deficiency and $13.5 \%$ had iron deficiency anemia. Also, $62.5 \%$ had mild hemoglobin level and $37.5 \%$ had moderate- severe mild hemoglobin level.

Conclusion:

The results indicate that, iron deficiency, hypertension can be seen in hypothyroidism during the 1st half of pregnancy. Hence, routine thyroid monitoring, checkup and appropriate management is necessary for hyperthyroidism in the 1 st half of pregnancy to avoid more adverse outcome.

\section{Introduction}

Among women of childbearing age, thyroid disorder is especially severe. Estimates indicate a degree of hypothyroidism among 2.5 percent of all pregnant people. The second most prevalent endocrine disorder in pregnancy is thyroid dysfunction. ${ }^{1}$ Maternal thyroid function changing activity during pregnancy result in thyroid disease and poor tolerance to these shifts. During pregnancy, maternal thyroid function changes and inadequate adaptation to these changes causes thyroid dysfunction.

Such changes are the result of multiple causes such as an increase in thyroglobulin because of elevated estrogen and human Chorionic gonadotrophin, decreased iodine losses from renal disorders attributable to the growth of glomerular filtration, modifications in peripheral metabolism of maternal thyroid hormone and alterations in iodine transfer to placenta. During pregnancy, the development of thyroid 
hormone and iodine rises by $50 \%$. In people with low thyroid reserve or iodine deficiency, pregnancy is a stress check for thyroid, which contributes to hypothyroidism. ${ }^{2}$

In pregnancy thyroid dysfunction was linked with adverse obstetric and fetal outcome. Most of these complications are miscarriage, anaemia, hypertension, preeclampsia, placental abruption, preterm labor, congestive heart failure, postpartum hemorrhage and the fetal complications are prematurity, birth asphyxia, low birth weight, low Apgar score, still birth, perinatal death, increased neonatal respiratory distress and decreased childhood cognitive function. ${ }^{3}$

Therefore, thyroid dysfunction (sub-clinical or overt) during early pregnancy is of utmost importance for clinician to detect thyroid dysfunction. 4

A safe and accurate examination of thyroid function during the pregnancy is thus important, since maternal thyroid deficiency can influence maternal safety, fetal well-being and obstetric results. ${ }^{5}$

The only way of evaluating the pathophysiologic features of thyroid dissolution during pregnancy, which can also detect minor thyroid abnormalities, is the detection of the thyroid function by using serum TSH during pregnancy. ${ }^{4}$

Therefore, early pregnancy screening for people at high risk is mandatory. But we would lose $4 \%$ of patients that we could have diagnosed and treated earlier if we only scan the community with a high risk. It is thus important for all pregnant women to be checked for compulsory in the first trimester of pregnancy. ${ }^{6}$

The objective of the study was to assess the relationship of multiple risk factors and thyroid functions among Bangladeshi young women during $1^{\text {st }}$ half of the pregnancy.

\section{Materials And Methods}

This cross-sectional study was done in in the Department of Obstetrics \& Gynecology of Dhaka Medical College Hospital, Dhaka on 200 pregnant women in their $1^{\text {st }}$ half of pregnancy attending antenatal clinic, DMCH from December 2018 to December 2019. Where inclusion criteria were healthy singleton pregnant women up to 20 weeks of gestation. On the other hand, exclusion criteria were multiple pregnancy, known cases of thyroid disorder, known metabolic disorders like DM \& HTN, cute febrile illness, patient taking drugs that interfere with thyroid function like lithium, amioderone, steroids, carbamazepine, dopamine and salicylates.

With aseptic precaution $5 \mathrm{ml}$. of venous blood drawn from each patient and taken into a sterile test tube which kept in standing position up to full formation of clot then serum was separated and taken into two eppendorf's. After that send to the laboratory for measurement of serum $\mathrm{TSH}^{-\mathrm{FT}_{3}}$ and $\mathrm{FT}_{4}$. Serum sample will be immediately assayed for the levels of serum TSH and serum samples were stored at $4^{\circ} \mathrm{C}$ for levels of $\mathrm{FT}_{3}$ and $\mathrm{FT}_{4}$. However, the serum $\mathrm{FT}_{3}$ and $\mathrm{FT}_{4}$ were estimated only in those women who 
exhibit abnormal serum TSH levels. Estimation was carried out by ADIVA centaur CP and Radioimmunoassay.

Written consent was taken from those who agreed to participate in the study. Date were collected in a structured questionnaire by the history taking, clinical examination and biochemical investigations.

Data analysis: Data were processed and analyzed using SPSS (statistical Package for social sciences), window version 21.0. The test statistics was used to analyze the data are chi- square $\left(x^{2}\right)$ test and unpaired student's t-test with its 95\% confidence interval (CI). For all analytical tests, the level of significance will be set at 0.05 and $p$ value of $<0.05$ will be considered statistically significant. Ethical clearance was obtained from appropriate authority. Privacy and confidentiality were strictly maintained.

\section{Results}

The demographic characteristics of 200 thyroid pregnant women in the first half of pregnancy where, most of the patients belong to 20-25 years age group. From euthyroid group $87.8 \%$ patients completed their graduation where as in subclinical and overt hypothyroidism group it was $4.9 \%$ and $7.3 \%$. From subclinical and overt hypothyroidism group 6.3\% pregnant women were underweight and $1.4 \%, 15.3 \%$ patients were overweight. The following table is given below in detail:

\section{Table1. Thyroid function status according to demographic characteristics among 200 thyroid pregnant women in the first half of pregnancy}

\begin{tabular}{|c|}
\hline 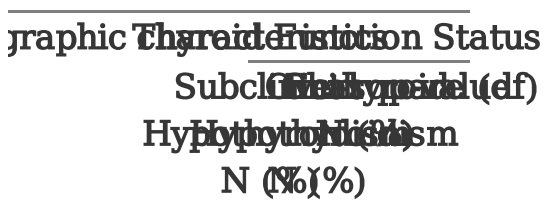 \\
\hline 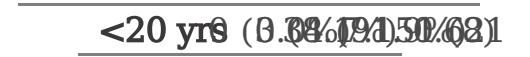 \\
\hline 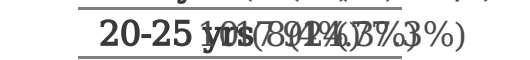 \\
\hline$\overline{>25}$ yrs (4.89o(86).4\%) \\
\hline ationHouselwife(22) \\
\hline 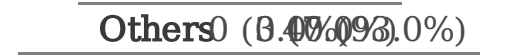 \\
\hline 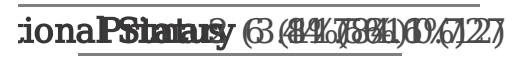 \\
\hline 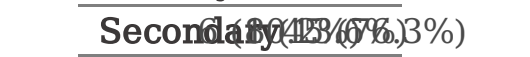 \\
\hline 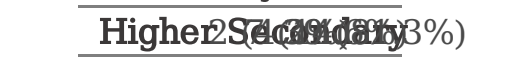 \\
\hline Graduaté2. (18/B8ס).8\%) \\
\hline 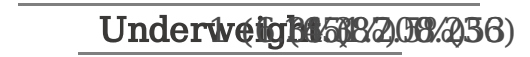 \\
\hline NormaD1(289)ex.890\%\%) \\
\hline Overweilgh( $19 x(83 \% \%)$ \\
\hline
\end{tabular}

*BMI range: $<20.5=$ underweight; 20.6-24.9 = normal; $>25$ = overweight

In table-2 shows association and correlation of obstetric characteristics and genetic inheritance according to thyroid function status, where there were no significant correlations were shown among 
gestation age, gravida and thyroid functional status. Where genetic inheritance was strongly associated with thyroid status and abnormally. The following table is given below in detail:

Table 2. Association and correlation of obstetric characteristics and genetic inheritance according to thyroid function status among thyroid pregnant patients in the first half of trimester $(n=200, d f=4)$

\begin{tabular}{|c|c|}
\hline \multicolumn{2}{|c|}{ 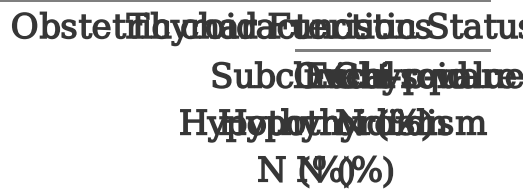 } \\
\hline \multicolumn{2}{|c|}{ 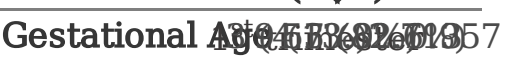 } \\
\hline & 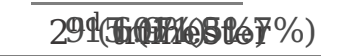 \\
\hline \multirow[t]{2}{*}{ Gravida } & 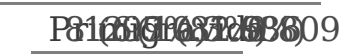 \\
\hline & 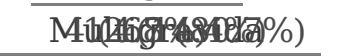 \\
\hline \multicolumn{2}{|c|}{ Genetic Inhefithn } \\
\hline & 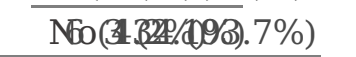 \\
\hline
\end{tabular}

In table-3 shows status of hemoglobin level, iron deficiency and blood pressure of thyroid pregnant patients where the rate of iron deficiency, elevated blood pressure and impaired hemoglobin level were strongly correlated with thyroid dysfunction. The following table is given below in detail:

Table 3. Status of hemoglobin level, iron deficiency and blood pressure of thyroid pregnant patients in the first half of pregnancy $(n=200)$

\begin{tabular}{|c|c|}
\hline \multicolumn{2}{|c|}{ 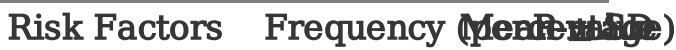 } \\
\hline Hemoglobluildvel & $125(6238 \%$ (19.032) \\
\hline \multicolumn{2}{|c|}{ 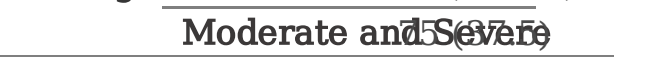 } \\
\hline Iron deficisncynal & $161(8065 \%$ - 0.000 \\
\hline Mild & $12(6 \%)$ \\
\hline \multicolumn{2}{|c|}{ Advanced 27 (13.5\%) } \\
\hline \multicolumn{2}{|c|}{ Blood Preskmeal $165(8276 \%$ / } \\
\hline \multicolumn{2}{|c|}{ Stage-1 Hypentension) } \\
\hline \multicolumn{2}{|c|}{ Stage-2 Hypentensiøn } \\
\hline
\end{tabular}

In table-4 shows comparison of thyroid function among different groups according to iron status where serum TSH concentrations were higher and FT4 concentrations were lower in IDA group than Mid iron deficiency and normal group. The following table is given below in detail:

Table 4. Comparison of thyroid function among different groups according to iron status $(n=200)$

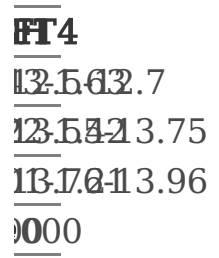

푸

133-5.63.7

12.5.2.23.75

1B.1.6-113.96

$\overline{\mathbf{0 0 0} 0}$

*IDA= iron deficiency anemia; $M I D=$ mild iron deficiency; $N C=$ normal control; $T S H=$ thyroid stimulating hormone; FT4= free thyroxine 
TSH $(\mathrm{m} / \mathrm{U} / \mathrm{L})=0.3-4.6\left(1^{\text {st }}\right.$ trimester $)$, Free T4 $(\mathrm{pmol} / \mathrm{L})=12.1-19.6\left(1^{\text {st }}\right.$ trimester $)$

\section{Discussion}

This cross-sectional study has been performed over 200 pregnant women in their $1^{\text {st }}$ half of pregnancy attending antenatal clinic. In this study, the risk factors those were comparatively higher in correlation with thyroid disfunction, were hemoglobin level, iron deficiency, genetic inheritance, blood pressure, TSH and FT4 level.

Moreover, according thyroid status and their 1 st half of the pregnancy we divided patients into three group. We found that most of the patients belong to $20-25$ years age group, where $8.4 \%$ belong subclinical hypothyroidism group, $14.3 \%$ were hypothyroidism and $77.35 \%$ patients were euthyroid group. In a study in Poland, $10.4 \%$ of the pregnant women screened for thyroid dysfunction in their 1 st trimester exhibited (hypothyroidism), which is quite close to this study findings. ${ }^{7}$ In a similar study conducted in Bangladesh where, clinical and subclinical hypothyroidism together comprised $8 \%$ of the pregnant women and clinical and subclinical hyperthyroidism together formed $7.5 \%$ thus constituting a total $15.5 \%$ with abnormal thyroid function which compares well with the findings of the present study. ${ }^{4}$ According to other report, in contrast to hyperthyroidism, hypothyroidism is quite common in pregnancy, which correlated with this study where subclinical hypothyroidism was $13 \%$ in comparison to subclinical hyperthyroidism(3\%). ${ }^{8}$ However, in another study of screening for subclinical hypothyroidism in pregnant women demonstrated a much lower proportion of hypothyroid status (1 in 40 antenatal mothers screened). ${ }^{9}$

According to our study, we didn't find any association between age and thyroid function alteration. Which is supported by one study. ${ }^{4}$

In this study neither gravidity of women nor their gestational age was found to be associated with their thyroid function status. While an Indian study reported no variations in serum TSH and serum FT3 between trimesters, but there is a significant variation in FT4 between trimesters with values decreasing with advancing gestational age. ${ }^{10}$

But we found that genetic inheritance is significantly associated with thyroid dysfunction. Where $10.5 \%$ subclinical hypothyroidism group and $36.8 \%$ from overt hypothyroidism patients had family history of thyroid dysfunction. In one study said that, autoimmune thyroid disease commonly runs in families, and the search for genes which increase susceptibility has identified several good candidates, particularly those involved in immune regulation and thyroid function. ${ }^{11}$

From our study, we also found that the rate of iron disfunction strongly correlated with thyroid dysfunction. $6 \%$ patients had mid-level of iron deficiency and $13.5 \%$ had iron deficiency anemia. Also, 
62.5\% had mild hemoglobin level and 37.5\% had moderate- severe mild hemoglobin level. Lower hemoglobin level is significantly associated with thyroid dysfunction too. In one report said that, Hemoglobin levels of less than $11 \mathrm{~g} / \mathrm{dL}$ at any time during pregnancy are considered abnormal. Once anemia is recognized, the possibility of iron deficiency should be considered. ${ }^{12}$

Up to $52 \%$ of pregnant women in the developing world are affected. ${ }^{13}$ Lowered iron stores in their newborn baby will increase the risk of subsequent iron deficiency anemia. Prematurity and early weaning off breastfeeding increases the risk further, because of reduced iron stores. ${ }^{14}$

Hypertension is the world's leading preventable risk for early death and disability, affecting $26.4 \%$ of the world's adult population. ${ }^{15-16}$ Apart from the majority of patients with main (essential) elevated blood pressure, the secondary hypertension subgroup of $\sim 10 \%$ is affected. Among the underlying diseases several are of endocrine origin and thyroidal impairments represent an even smaller percentage of the secondary hypertension cases; their incidence and form of presentation varies with age and studied population. ${ }^{17}$ Thyroid dysfunctions, both hypo- and hyperthyroidism may increase the risk of hypertension. ${ }^{18-19}$ In our study we found similar type of result where $6.5 \%$ had stage- 1 hypertension and $11 \%$ patients had stage- 2 hypertension. Also, abnormal blood pressure level is highly correlated with thyroid dysfunction status.

Another article mentioned that, Hypothyroid patients have other atherosclerotic cardiovascular disease risk factors and an apparent increase in risk of stroke as well. ${ }^{20-21}$ The blood pressure changes, alterations in lipid metabolism, decreased cardiac contractility, and increased SVR that accompany hypothyroidism are caused by decreased thyroid hormone and action on multiple organs such as the heart, liver, and peripheral vasculature and are potentially reversible with thyroid hormone replacement. ${ }^{22}$ In present study serum FT4 concentrations were lower and TSH concentrations were higher in subjects with iron deficiency, which quite similar to other studies. ${ }^{23-24}$ Also, in our study changes of FT4 and TSH level were highly correlated with thyroid dysfunction.

\section{Conclusion}

From the findings of the study and discussion thereof it can be concluded that almost one and half (1.53) in every ten overweight women may have thyroid disorder (overt hypothyroidism) in the first half of pregnancy. Besides, there can be a link between family history and thyroidism because around three and half in every ten-patient had family history of thyroid dysfunction. None of the rest demographic and obstetric characteristics plays any role in the development of abnormality in thyroid function. Euthyroid hypothyroidism is far more common than the subclinical and overt hyperthyroidism. The proportion of iron deficiency, hemoglobin and hypertension is strongly correlated with thyroid dysfunction too. So, if feasible routine screening at least serum TSH level can be done in all pregnant women attending antenatal visit. Routine thyroid monitoring, checkup and appropriate management is necessary for hyperthyroidism and especially in the very $1^{\text {st }}$ half of pregnancy to avoid more adverse outcome 


\section{Declarations}

Ethical clearance was obtained from appropriate authority. Privacy and confidentiality were strictly maintained.

Conflict of Interest: All authors declare that they have no competing interests.

Funding Agency: None to declare

\section{References}

1. Konin, S. S., \& Bhinder, G. K. (2013). Detection of thyroid functions in early pregnancy as a universal screening. Journal of Evolution of Medical and Dental Sciences, 2(49), 9457-9466.

2. Saraladevi, R., Nirmala Kumari, T., Shreen, B., \& Usha Rani, V. (2016). Prevalence of thyroid disorder in pregnancy and pregnancy outcome. IAIM, 3(3), 1-11.

3. Krishnamma, B., Prabhavathi, V., \& Prasad, D. K. V. (2017). Prevalence of thyroid dysfunction in pregnant women and the need for universal screening: an observational study in Northern Andhra Pradesh population. International Journal of Reproduction, Contraception, Obstetrics and Gynecology, 6(6), 2537.

4. Tania, S. N., \& Islam, F. (2014). Screening of Thyroid Function in the First Half of Pregnancy. Bangladesh Journal of Obstetrics \& Gynaecology, 29(1), 26-31.

5. Krassas, G. E., Poppe, K., \& Glinoer, D. (2010). Thyroid function and human reproductive health. Endocrine reviews, 31(5), 702-755.

6. Dave, A., Maru, L., \& Tripathi, M. (2014). Importance of Universal screening for thyroid disorders in first trimester of pregnancy. Indian journal of endocrinology and metabolism, 18(5), 735.

7. Matuszek, B., Zakoscielna, K., Baszak-Radomanska, E., Pyzik, A., \& Nowakowski, A. (2011). Universal screening as a recommendation for thyroid tests in pregnant women. Annals of agricultural and environmental medicine, $18(2)$.

8. Klein, R. Z., Haddow, J. E., Falx, J. D., Brown, R. S., Hermos, R. J., Pulkkinen, A., \& Mitchell, M. L. (1991). Prevalence of thyroid deficiency in pregnant women. Clinical endocrinology, 35(1), 41-46.

9. Spong, C. Y. (2005). Subclinical hypothyroidism: should all pregnant women be screened?. Obstetrics \& Gynecology, 105(2), 235-236.

10. Marwaha RK, Chopra S, Gopalakrishnan S, Sharma B, Kanwar RS, Sastry A. Establishment of reference range for thyroid hormones in normal pregnant Indian women. BJOG 2008; 115(5):602-6.

11. Panicker, V. (2011). Genetics of thyroid function and disease. The Clinical Biochemist Reviews, 32(4), 165.

12. Shill, K. B., Karmakar, P., Kibria, M. G., Das, A., Rahman, M. A., Hossain, M. S., \& Sattar, M. M. (2014). Prevalence of iron-deficiency anaemia among university students in Noakhali region, Bangladesh. Journal of Health, Population, and Nutrition, 32(1), 103 
13. Sato, A. P. S., Fujimori, E., Szarfarc, S. C., Borges, A. L. V., \& Tsunechiro, M. A. (2010). Food consumption and iron intake of pregnant and reproductive aged women. Revista Latino-Americana de Enfermagem, 18(2), 247-254.

14. Abu-Ouf, N. M., \& Jan, M. M. (2015). The impact of maternal iron deficiency and iron deficiency anemia on child's health. Saudi medical journal, 36(2), 146.

15. Kearney, P. M. (2005). reynolds K, Munter P, Whelton PK, He J. Global burden of hypertension: analysis of worldwide data. Lancet, 365, 217-23.

16. GBD 2013 Risk Factors Collaborators. (2015). Global, regional, and national comparative risk assessment of 79 behavioural, environmental and occupational, and metabolic risks or clusters of risks in 188 countries, 1990-2013: a systematic analysis for the Global Burden of Disease Study 2013. Lancet (London, England), 386(10010), 2287.

17. Charles, L., Triscott, J., \& Dobbs, B. (2017). Secondary hypertension: discovering the underlying cause. American family physician, 96(7), 453-461.

18. Cappola, A. R., \& Ladenson, P. W. (2003). Hypothyroidism and atherosclerosis. The Journal of Clinical Endocrinology \& Metabolism, 88(6), 2438-2444.

19. Prisant, L. M., Gujral, J. S., \& Mulloy, A. L. (2006). Hyperthyroidism: a secondary cause of isolated systolic hypertension. The Journal of Clinical Hypertension, 8(8), 596-599.

20. Danzi, S., \& Klein, I. (2003). Thyroid hormone and blood pressure regulation. Current hypertension reports, 5(6), 513-520.

21. Franklyn, J. A., Sheppard, M. C., \& Maisonneuve, P. (2005). Thyroid function and mortality in patients treated for hyperthyroidism. Jama, 294(1), 71-80.

22. Palmieri, E. A., Fazio, S., Lombardi, G., \& Biondi, B. (2004). Subclinical hypothyroidism and cardiovascular risk. Treatments in endocrinology, 3(4), 233-244.

23. Yu, X., Shan, Z., Li, C., Mao, J., Wang, W., Xie, X., .. \& Xu, B. (2015). Iron deficiency, an independent risk factor for isolated hypothyroxinemia in pregnant and nonpregnant women of childbearing age in China. The Journal of Clinical Endocrinology \& Metabolism, 100(4), 1594-1601.

24. Brigham, D. E., \& Beard, J. L. (1995). Effect of thyroid hormone replacement in iron-deficient rats. American Journal of Physiology-Regulatory, Integrative and Comparative Physiology, 269(5), R1140R1147.

25. 